\title{
Review Article Ultrasound in Dengue: A Scoping Review
}

\author{
Neelesh Dewan, ${ }^{1,2,3 \star} \dagger$ Daniela Zuluaga, ${ }^{2} \dagger$ Lyda Osorio, ${ }^{2}$ Mary-Ellen Krienke, ${ }^{3}$ Caitlin Bakker, ${ }^{4}$ and Jonathan Kirsch ${ }^{5}$ \\ ${ }^{1}$ University of Miami Miller School of Medicine, Miami, Florida; ${ }^{2}$ Epidemiology and Population Health Research Group (GESP), School of Public \\ Health, Universidad del Valle, Cali, Colombia; ${ }^{3}$ University of Minnesota Medical School, Minneapolis, Minnesota; ${ }^{4}$ University of Minnesota, Health \\ Sciences Libraries, Minneapolis, Minnesota; ${ }^{5}$ Section of Hospital Medicine, Division of General Internal Medicine, University of Minnesota Medical \\ School, Minneapolis, Minnesota
}

\begin{abstract}
To improve dengue triage and treatment decisions, the WHO recommends classifying the disease as dengue with or without warning signs or severe dengue. Vascular leakage is a key component of the pathophysiology of severe dengue and is detectable by ultrasound. The objective of this scoping review is to describe the primary literature on the use of ultrasound in dengue, summarize the findings, and identify knowledge gaps. Our database search retrieved 1,489 records which were reduced to 177 studies following eligibility screening. Descriptive analyses were conducted. Results showed most studies are from South Asia $(n=92,52 \%)$ and assessed hospitalized dengue patients $(130,82 \%)$. Radiologists were the most reported ultrasonographers (14, 8\%), and conventional ultrasound (39, 68\%) was preferred over portable $(9,16 \%)$. The most common ultrasound findings in dengue were ascites (107, 60\%), pleural effusion (102, $58 \%)$, and gallbladder wall thickening $(97,55 \%)$. However, the lack of a standard protocol to perform the ultrasound examination in dengue patients hinders conclusions about the frequency of ultrasound findings in dengue. Given the progress of current ultrasound technology, a focused point-of-care ultrasound protocol for early detection of vascular leakage in dengue is needed to generate the evidence required for its implementation in routine care.
\end{abstract}

\section{INTRODUCTION}

More than half the world's population is at risk for dengue infection due to the expansive range of the Aedes aegypti mosquito, with approximately 390 million cases a year. ${ }^{1,2}$ The clinical course of dengue infection is unpredictable. Although most cases resolve spontaneously, a significant minority can lead to shock, severe hemorrhage, organ involvement, and death. Therefore, the 2009 WHO guidelines recommend classifying disease into dengue without warning signs, dengue with warning signs, or severe dengue for prompt and proper clinical management. ${ }^{3,4}$

A systemic vascular leakage syndrome is central to the clinical course of severe dengue..$^{5}$ In routine care, clinical signs such as the presence of fluid accumulation (e.g., pleural effusion and ascites), hypotension, and narrowing pulse pressure, or laboratory evidence of hemoconcentration or hypoproteinemia can detect vascular leakage, but it can be challenging and easily missed.

Chest X-rays can aid in the detection of pleural fluid, but poster-anterior radiographs are only able to detect $>200 \mathrm{~mL}$ of fluid and lateral radiographs only $>50 \mathrm{~mL}$; chest ultrasound, on the other hand, can detect as little as $5-50 \mathrm{~mL}$ of pleural fluid with $100 \%$ sensitivity. ${ }^{6}$ Furthermore, ultrasound has been demonstrated to identify pleural effusions even before changes in hematocrit levels. ${ }^{7}$

Ascites is a common manifestation of the vascular leakage syndrome seen in severe dengue, and abdominal ultrasound has been deemed the first-line imaging method to study ascites. ${ }^{8}$ WHO guidelines state that abdominal ultrasound can be used for diagnosis of vascular leakage in dengue; however, insufficient evidence exists regarding its performance and prognostic value. ${ }^{4,9}$

\footnotetext{
*Address correspondence to Neelesh Dewan, University of Miami Miller School of Medicine, 9806 Stonebridge Way, Mint Hill, NC 28227. E-mail: neeleshdewan92@gmail.com

$\dagger$ These authors contributed equally to this work.
}

In addition to the mentioned pleural and abdominal effusions, other sonographic signs also manifest in suspected or confirmed dengue patients. ${ }^{10}$ The objective of this study is to describe the scope of ultrasound use and findings in dengue, with the aim of informing medical professionals, policy decision-makers, and researchers on knowledge gaps where further research is needed. This is particularly relevant in the light of current progress in portable ultrasound technology easily available in primary care settings and emergency wards in both dengue-endemic and non-endemic countries.

\section{MATERIALS AND METHODS}

Study design and search strategy. We conducted a scoping review to determine the extent of ultrasound use for any purpose in the context of dengue. The search strategy was developed using both natural language and controlled vocabulary for "ultrasound" and "dengue." This search was executed across 10 databases: Medline and Embase via Ovid, Medline via PubMed, Cochrane Library via Wiley, Global Index Medicus, ClinicalTrials.gov, WHO International Clinical Trials Registry, WHO Library Database, Opengrey.eu, and Scopus. To facilitate the capture of the broadest range of literature, no limitations were placed on the language of publication, study design, or year of publication. The complete search strategy is available in Appendix 1, and the protocol for this review was registered before initiation through Open Science Framework. ${ }^{11}$ Retrieved citations were compiled and de-duplicated using EndNote X7 (Clarivate Analytics 2013), and exported to Rayyan (Qatar Computing Research Institute, Doha, Qatar) for screening. ${ }^{12-14}$

Study selection. Title and abstract screening were completed by two independent reviewers based on the previously defined inclusion and exclusion criteria. Items were included if they used ultrasound and involved persons with the diagnosis of dengue and were excluded if they did not report findings from ultrasound and did not report original data, such as editorials and review articles. The full-text screening phase was 
again undertaken by two independent reviewers. Where there were conflicts between the two reviewers, consensus was reached through discussion. Articles written in English, Spanish, French, and Portuguese were included, reflecting the reviewers' language abilities. Three were written in Vietnamese and were not able to be reliably translated, and thus were not included.

Data extraction and analysis. A data extraction form was developed using REDCap (Research Electronic Data Capture, Vanderbilt University, Nashville, TN) hosted at the University of Minnesota and piloted before implementation for further refinement. ${ }^{15}$ Two independent reviewers extracted data regarding study type, setting, patient demographics, dengue clinical and laboratory-based methods, ultrasound equipment, ultrasound examination method, outcome measurements, and adverse events. The reviewers resolved discrepancies through consensus, or, where necessary, through the intervention of a third reviewer. Although risk of bias assessment is not a required component of scoping reviews, it was felt that this step was necessary to capture the strengths and weaknesses of the included literature. Critical appraisal of manuscripts was performed using the QUADAS-2 tool (University of Bristol, Bristol, United Kingdom). ${ }^{16}$ Descriptive analyses were carried out in STATA 10 (StataCorp. 2007, College Station, TX); median and range were estimated for quantitative data, and absolute and relative frequencies were determined for categorical data. The anatomical sites of ultrasound examination were categorized into four groups: 1) thorax, 2) abdomen, 3) cardiac, and 4) other. Thorax variables included pulmonary B-lines and pleural effusion (right-sided, left-sided, or bilateral); abdomen variables included ascites (hepatorenal, splenorenal, and/or pelvic), gallbladder edema or wall thickening, inferior vena cava collapsibility, splenomegaly, hepatomegaly, and hepatosplenomegaly; cardiac variables included pericardial effusion, hypokinesia, reduced ejection fraction, and ventriculomegaly; and "other" referred to findings other than those mentioned earlier.

\section{RESULTS}

Characteristics of included studies. A total of 1,489 records were identified through our database search. After removal of duplicates, 829 records remained. From these, 597 records were excluded during title and abstract screening. Fifty-five records from the remaining 232 were excluded during full-text screening (10 full-texts were not available), resulting in 177 studies for inclusion in our analyses (Figure 1). The included studies were published from 1989 to 2018 and conducted mostly in India $(n=56,32 \%)$, followed by Sri Lanka $(n=20,11 \%)$, Pakistan $(n=16,9 \%)$, Indonesia $(n=15,8 \%)$,
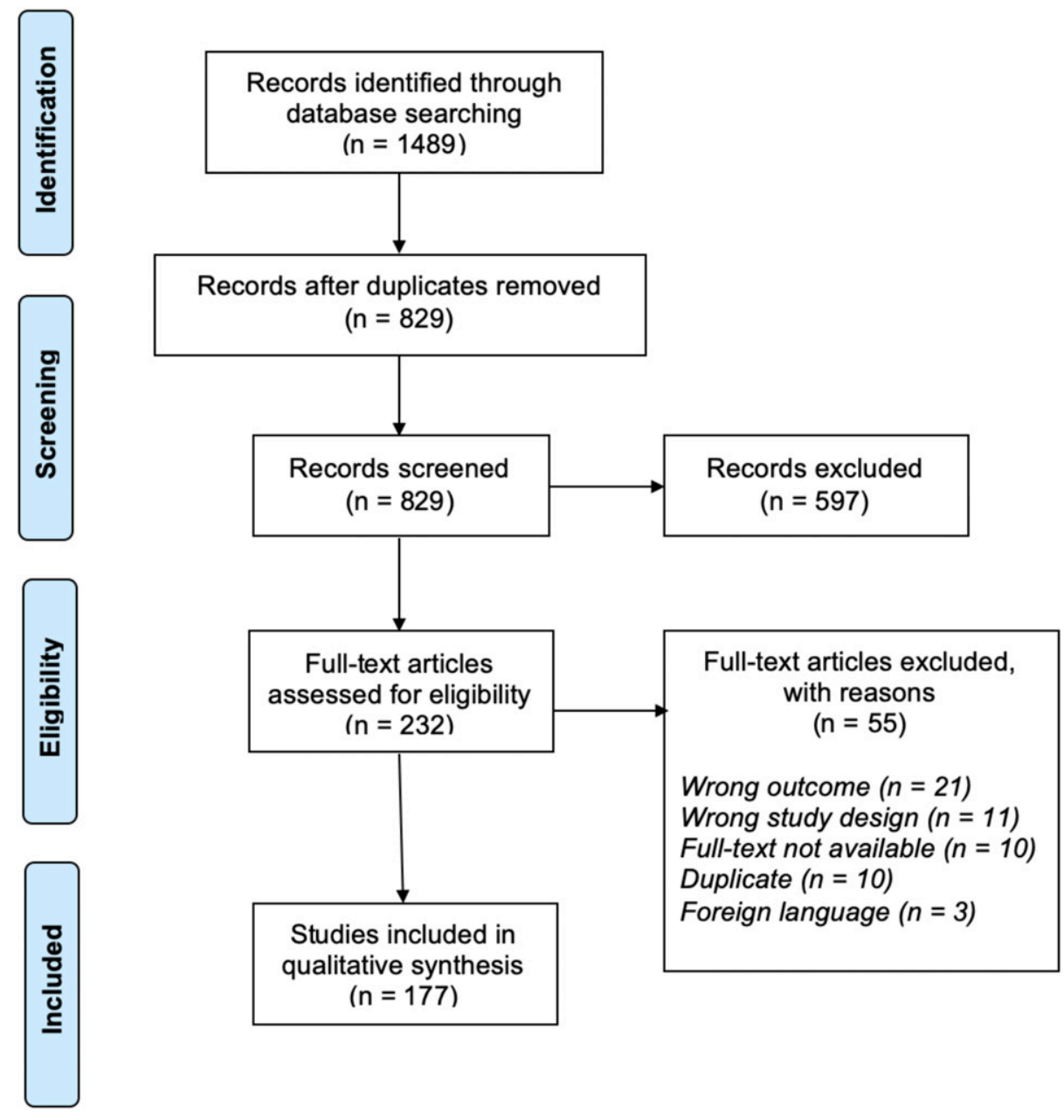

FIGURE 1. Flowchart of study selection process. This figure appears in color at www.ajtmh.org. 
and Thailand ( $n=13,7 \%)$. In the Americas, Brazil ( $n=10,29 \%)$ and Cuba ( $n=8,23 \%$ ) contributed to most of the studies (Figure 2). ${ }^{17}$

In relation to study type, 91 studies (51\%) were prospective, 27 (15\%) retrospective, 40 (23\%) case reports, 12 (7\%) crosssectional, and seven (4\%) case series. The median study sample size was 55 (range 1-1782), and of the 23 studies that mentioned their sampling method (13\%), 10 (43\%) were consecutive, seven (30\%) by convenience, and six (26\%) random. Of the studies that reported the clinical setting of included patients ( $n=158,89 \%)$, a majority assessed hospitalized patients ( $n=130,82 \%), 20(13 \%)$ intensive care patients, and eight (5\%) ambulatory patients. Children (patients $\leq 18$ years old) were included in 102 studies (58\%). Most studies that included children were performed in Asia ( $n=84,82 \%)$, followed by South America ( $n=8,8 \%$ ), Central America and the Caribbean ( $n=8,8 \%)$, and North America $(n=2,2 \%)$. None of the studies that included children were from Europe or Oceania. Only seven studies (4\%) included pregnant women. Risk of bias and applicability concerns were found to be low in most of the studies. When risk of bias was found to be unclear or high, it was principally in patient selection and the index test (i.e., ultrasound) (Figure 3).

Dengue identification, confirmation, and classification. Almost half of the studies ( $n=86,48 \%$ ) used only clinical criteria to identify eligible participants, a quarter $(n=42,24 \%)$ used laboratory methods (based on NS1, IgM/lgG, or reverse transcription PCR) only, and the rest used both clinical and laboratory criteria ( $n=49,28 \%$ ). The most frequent method to confirm dengue infection was laboratory testing ( $n=145,82 \%)$. Ten studies (6\%) used clinical criteria alone to confirm cases. Confirmation based on both clinical criteria and laboratory testing occurred in 19 studies (11\%). Three studies (2\%) did not specify how they confirmed cases. Not all studies specified which WHO dengue case classification criteria (1997 or 2009) was used ( $n=58,33 \%)$. More studies used the 1997 criteria $(n=$ $32,55 \%)$ than the 2009 criteria $(n=26,45 \%)$. Two studies $(1 \%)$ considered both systems when classifying cases. In the hospitalized setting, 27 studies (55\%) relied on the 1997 criteria and 22 (45\%) on the 2009 criteria. Mortality outcomes were described in 51 studies (29\%), and in $61 \%(n=31)$ of these studies, death of at least one study participant occurred.

Ultrasound equipment and timing of examination. The level of training of the ultrasonographer was reported in 35 studies (20\%); 14 (40\%) reported a radiologist, eight (23\%) "the study researcher" without a description of their qualifications, four (11\%) a technician only, two (6\%) a technician or radiologist, and one each a cardiologist, "general physician or radiologist," "radiology resident or technician," pediatric intensivist, pediatrician, unspecified physician, and non-radiologist (3\%). Ultrasound equipment was described in 57 studies (32\%). Most of these studies used conventional, non-portable ultrasound equipment ( $n=39,68 \%$ ), whereas nine studies (16\%) used portable ultrasound equipment. The other nine studies (16\%) provided limited information on the ultrasound equipment that was indeterminable by reviewers to be conventional or portable. A portion of the studies ( $n=52,29 \%$ ) reported the number of ultrasound examinations per patient and the time interval to examination realization. In 19 studies (37\%), a single ultrasound examination per patient was performed, whereas in the remaining 33 studies (63\%), follow-up examinations were also performed. Timing of the first ultrasound was between days 1 and 7 relative to a reference point, which was either admission to the hospital ( $n=36,20 \%)$, beginning of the critical phase of disease $(n=11,6 \%)$, fever onset $(n=5,3 \%)$, or not specified $(n=$ $125,71 \%)$. Most primary ultrasound examinations $(n=33,63 \%)$ were performed on day 1 relative to the reference point.

Ultrasound examination and findings. The median number of anatomical sites examined per patient was three (range 1-6). The thorax was the most frequently examined site $(n=$

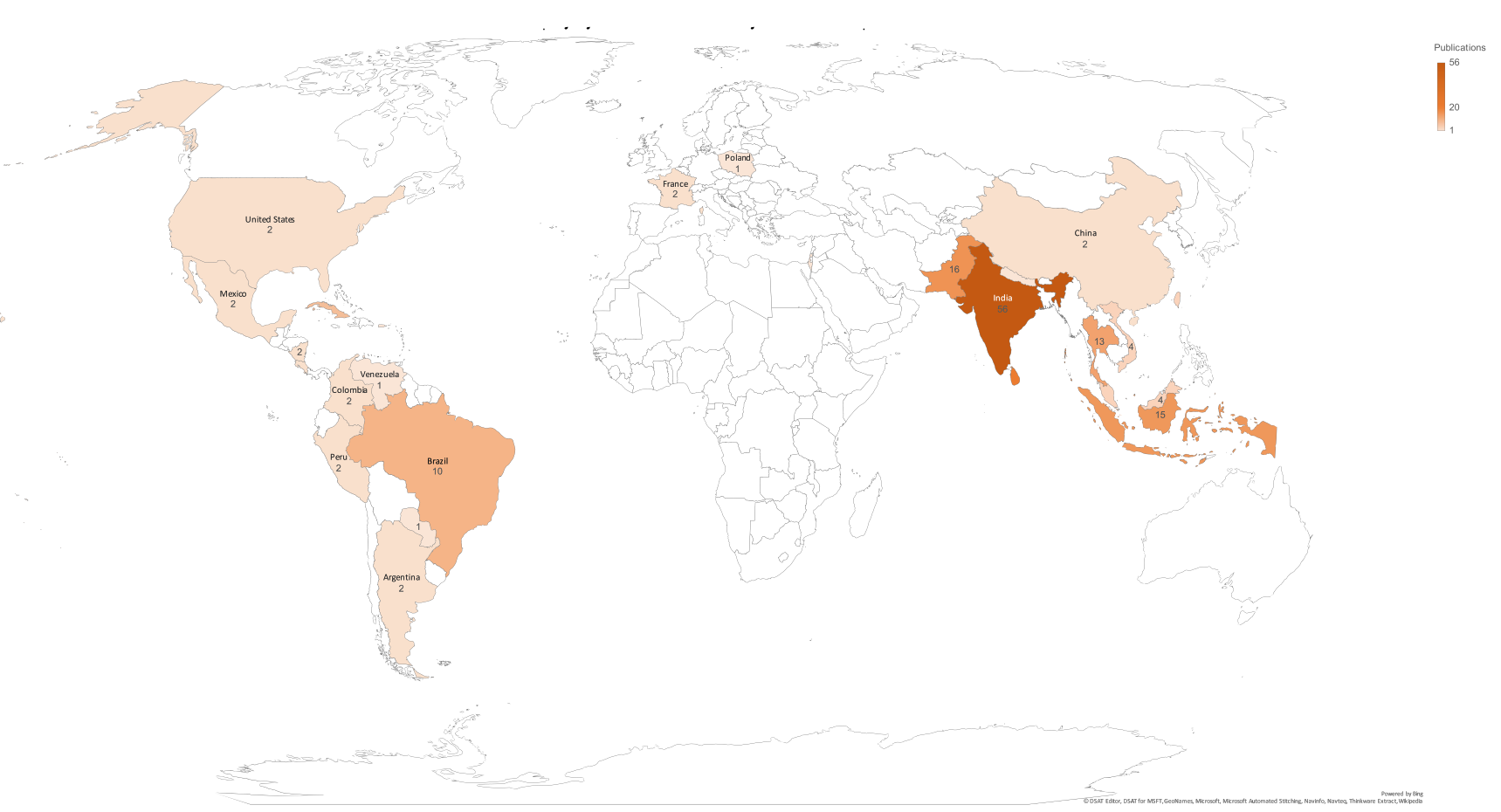

FIGURE 2. Heat map of countries with reported ultrasound use in dengue. ${ }^{18}$ This figure appears in color at www.ajtmh.org. 


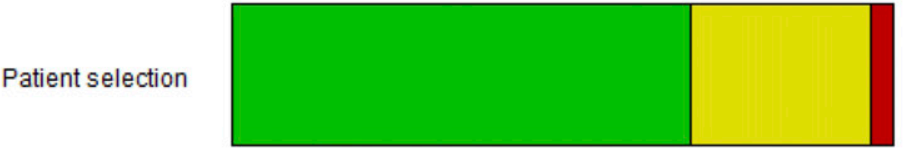

Index test

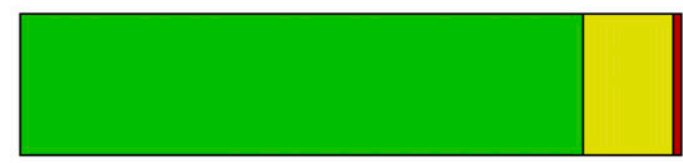

Reference standard
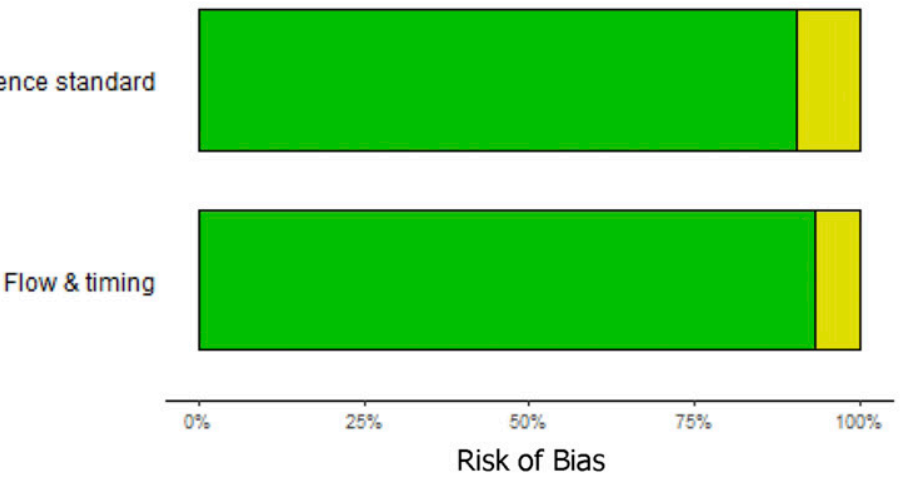
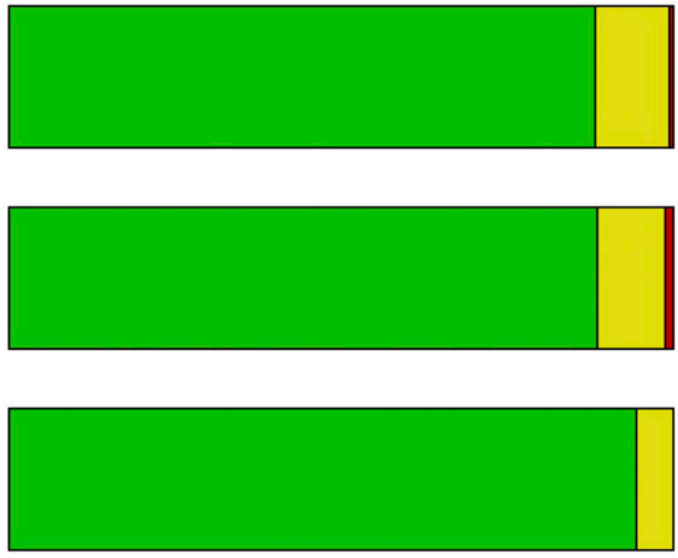

Low risk of bias

Some concerns

High risk of bias

FIGURE 3. Risk of bias and applicability concern of included studies organized by QUADAS-2 domain. This figure appears in color at www.ajtmh.org.

$102,57 \%)$, followed by the abdomen $(n=42,24 \%)$, heart $(n=$ $17,10 \%)$, and other parts of the body, such as the eyes, pancreas, intestines, knee joint, and psoas and abdominal wall muscles $(n=16,9 \%)$.

Thoracic ultrasound findings. The most commonly encountered thoracic ultrasound finding was pleural effusion ( $n=$ $102,58 \%)$. Laterality of effusion was specified in 44 studies (43\%); 10 (10\%) reported bilaterality, 16 (16\%) right-sided laterality, 18 (18\%) left-sided laterality, and 58 (57\%) did not specify. The presence of pulmonary B-lines as a sign of pulmonary edema was described in one study $(1 \%){ }^{18}$

Abdominal ultrasound findings. Of the studies that evaluated organomegaly ( $n=110,62 \%$ ), eight studies (7\%) found ultrasound evidence of hepatosplenomegaly, 53 (48\%) hepatomegaly, and $48(44 \%)$ splenomegaly. Few studies found the inferior vena cava collapsible $(n=4,2 \%)$. Most of the studies reported gallbladder wall thickening (GBWT, $n=97,55 \%$ ), but the cutoff for GBWT varied. The cutoff was not reported in a large number of studies ( $n=60,62 \%)$. Three millimeters was most often used ( $n=29,30 \%)$. Less common cutoffs were $5 \mathrm{~mm}(n=3,3 \%), 4 \mathrm{~mm}(n=1,1 \%)$, $3.5 \mathrm{~mm}(n=2,2 \%)$, and $2 \mathrm{~mm}(n=2,2 \%)$. One manuscript reported patterns of GBWT: a honeycomb pattern was seen in 21 patients (39\%, mainly severe dengue), a uniform echogenic pattern in 20 patients (37\%, mainly dengue with and without warning signs), striated in 11 (20\%), and asymmetric in two (4\%). ${ }^{19}$

Ascites was found in 107 (60\%) of the studies, and almost all of these studies ( $n=99,93 \%)$ reported unspecified ascites. Studies that specified the type of ascites found hepatorenal ( $n=6,6 \%)$ and pelvic ascites $(n=2,2 \%)$. Splenorenal ascites was not reported.

Cardiac ultrasound findings. Pericardial effusion was the most reported cardiac ultrasound finding ( $n=32,18 \%$ ), followed by reduced ejection fraction ( $n=23,13 \%)$, hypokinesia $(n=10,6 \%)$, and ventriculomegaly ( $n=5,3 \%)$. Pericardial effusion was reported as an isolated echocardiographic finding in 24 studies (75\%). In the remaining eight studies (25\%), pericardial effusion was associated with one or more other heart abnormalities.

Other ultrasound findings. Besides the thorax, abdomen, and heart, ultrasound findings were also reported in other anatomical sites. These included the eyes (bilateral narrow anterior chamber), pancreas (edema), small and large intestines (small bowel edema, jejunal-ileal and ileocecal intussusception, and edematous appendix), knee (joint effusion), and muscles (thickened psoas, rapidly expanding hematoma in the intramuscular plane of the right lower abdomen). ${ }^{20-34}$

Gold standard comparisons and outcome measurements. Comparison of ultrasound examinations against a gold standard was reported in only $9 \%(n=16)$ of studies. The majority $(n=10$, $63 \%$ ) used a radiologist as the gold standard, five (31\%) used a non-radiologist, and one used computed tomography (6\%). One study used the kappa index as an outcome measurement when comparing the main ultrasound performer with a gold standard. In this case, the sonographer was compared against another experienced sonographer, obtaining a kappa index of $>0.9 .^{35}$ Sensitivity and specificity of ultrasound to detect vascular leakage against a gold standard were not reported. Fifty articles (28\%) reported no ultrasound-related or study-related adverse events. The rest did not specify the presence or absence of adverse events (Table 1).

\section{DISCUSSION}

In this scoping review, we describe the current primary literature on the use of ultrasound in dengue to inform clinicians, 
TABLE 1

Select reported variables from included studies $(n=177)$ and their absolute $(n)$ and relative frequencies $(\%)$ or range, when applicable

\begin{tabular}{|c|c|c|}
\hline & Frequency & \\
\hline Reported variable & N & $\%$ \\
\hline Study characteristics & & \\
\hline Study type & & \\
\hline Prospective & 91 & 51 \\
\hline Retrospective & 27 & 15 \\
\hline Case report & 40 & 23 \\
\hline Case series & 7 & 4 \\
\hline Cross-sectional & 12 & 7 \\
\hline Median sample size (range) & 55 & $(1-1782)$ \\
\hline Setting $(n=158,89 \%)$ & & \\
\hline Ambulatory & 8 & 5 \\
\hline Hospitalized & 130 & 82 \\
\hline Intensive care & 20 & 13 \\
\hline Children included & & \\
\hline Yes & 102 & 58 \\
\hline No & 39 & 22 \\
\hline Did not specify & 36 & 20 \\
\hline Dengue criteria and mortality & & \\
\hline Patient identification & & \\
\hline Clinical criteria & 86 & 48 \\
\hline Laboratory methods & 42 & 24 \\
\hline Both & 49 & 28 \\
\hline Case confirmation & & \\
\hline Clinical criteria & 10 & 5 \\
\hline Laboratory methods & 145 & 82 \\
\hline Both & 19 & 11 \\
\hline Did not specify & 3 & 2 \\
\hline WHO classification system used ( $n=58,33 \%)$ & & \\
\hline 1997 & 32 & 55 \\
\hline 2009 & 26 & 45 \\
\hline Both & 2 & 1 \\
\hline Mortality $(n=51,29 \%)$ & & \\
\hline Yes & 31 & 61 \\
\hline No & 20 & 39 \\
\hline Ultrasound equipment and timing of examination & & \\
\hline Ultrasonographer level of training $(n=35,20 \%)$ & & \\
\hline Radiologist & 14 & 40 \\
\hline Study researcher & 8 & 23 \\
\hline Technician only & 4 & 11 \\
\hline "Technician or radiologist" & 2 & 6 \\
\hline Cardiologist & 1 & $3 \%$ \\
\hline "General physician or radiologist" & 1 & 3 \\
\hline "Radiology resident or technician" & 1 & 3 \\
\hline Pediatric intensivist & 1 & 3 \\
\hline Pediatrician & 1 & 3 \\
\hline Unspecified physician & 1 & 3 \\
\hline Non-radiologist & 1 & 3 \\
\hline Ultrasound equipment ( $n=57,32 \%)$ & & \\
\hline Conventional & 39 & 68 \\
\hline Portable & 9 & 16 \\
\hline Indeterminable & 9 & 16 \\
\hline Timing of ultrasound examination (relative to reference point) $(n=52,29 \%)$ & & \\
\hline Day 1 & 33 & 63 \\
\hline Days 2-7 & 19 & 37 \\
\hline Reference point for timing of ultrasound examination & & \\
\hline Fever onset & 5 & 3 \\
\hline Admission to hospital & 36 & 20 \\
\hline Beginning of critical phase & 11 & 6 \\
\hline Did not specify & 125 & 71 \\
\hline Ultrasound examination & & \\
\hline Median number anatomical sites examined per patient (range) & 3 & $(1-6)$ \\
\hline Anatomical sites examined & & \\
\hline Thorax & 102 & 57 \\
\hline Abdomen & 42 & 24 \\
\hline Cardiac & 17 & 10 \\
\hline Other (eyes, pancreas, intestines, knee joint, psoas, and abdominal wall muscles) & 16 & 9 \\
\hline Ultrasound findings & & \\
\hline Thoracic ultrasound findings & & \\
\hline Pleural effusion ( $n=102,58 \%)$ & & \\
\hline
\end{tabular}


TABLE 1

Continued

\begin{tabular}{|c|c|c|}
\hline & Frequency & \\
\hline Reported variable & N & $\%$ \\
\hline Right-sided & 16 & 16 \\
\hline Left-sided & 18 & 18 \\
\hline Bilateral & 10 & 10 \\
\hline Did not specify & 58 & 57 \\
\hline Pulmonary B-lines & 1 & 1 \\
\hline Abdominal ultrasound findings & & \\
\hline Organomegaly $(n=110,62 \%)$ & & \\
\hline Hepatomegaly & 53 & 48 \\
\hline Splenomegaly & 49 & 44 \\
\hline Hepatosplenomegaly & 8 & 7 \\
\hline Gallbladder wall thickening ( $n=97,55 \%)$ & & \\
\hline $5 \mathrm{~mm}$ & 3 & 3 \\
\hline $4 \mathrm{~mm}$ & 1 & 1 \\
\hline $3.5 \mathrm{~mm}$ & 2 & 2 \\
\hline $3 \mathrm{~mm}$ & 29 & 30 \\
\hline $2 \mathrm{~mm}$ & 2 & 2 \\
\hline Did not specify & 60 & 62 \\
\hline Ascites $(n=107,60 \%)$ & & \\
\hline Hepatorenal & 6 & 6 \\
\hline Splenorenal & 0 & 0 \\
\hline Pelvic & 2 & 2 \\
\hline Did not specify & 99 & 93 \\
\hline Cardiac ultrasound findings & & \\
\hline Pericardial effusion & 32 & 18 \\
\hline Reduced ejection fraction & 23 & 13 \\
\hline Hypokinesia & 10 & 6 \\
\hline Ventriculomegaly & 5 & 3 \\
\hline Gold standard comparisons & & \\
\hline Comparison against a gold standard $(n=16,9 \%)$ & & \\
\hline Radiologist & 10 & 63 \\
\hline Non-radiologist & 5 & 32 \\
\hline Computed tomography & 1 & 6 \\
\hline
\end{tabular}

public health authorities, and researchers. Summary and mapping of the current knowledge landscape in this manner serves to enhance identification and understanding of knowledge gaps. Findings show that ultrasound has been in use in dengue since the late 1980s, mainly in endemic countries such as India and in other south and Southeast Asian regions. This time line could be attributed to the patterns of dengue spread after 1970, the evidence on the use of ultrasound to detect subclinical ascites, the increased number of medical specialties using ultrasound, and the availability of ultrasound equipment worldwide, even in resource-limited settings. ${ }^{4,36-38}$ The geographical distribution could also reflect differences in access to ultrasound services for dengue patients.

In our assessment of potential bias in included studies, we focused on "the degree to which estimates of the diagnostic accuracy avoided risk of bias" and "the extent to which primary studies are applicable to the review's research question," where bias is considered to be the product of "systematic flaws or limitations in the design or conduct of a study that distort the results." 16 Risk of bias and applicability concerns were highest in the area of patient selection, which could result in an exaggeration or underestimation of results for a reported variable in our review. Where there were some concerns of risk of bias, this was indicative of incomplete reporting. We noted significant heterogeneity between studies with regard to reference standards and use of the index test; although this is not indicative of bias, it limits our ability to synthesize data from and draw conclusions based on the body of literature.
Included studies' reliance on the clinical criteria for the identification of participants may be a result of the expensive cost and technical demands of early laboratory diagnostic testing (i.e., viral isolation in cell culture, nucleic acid detection, or antigen detection), especially in developing nations. ${ }^{4}$ Given this dependence on clinical identification of dengue patients, the question arises whether researchers in these studies were truly assessing dengue patients. However, many studies considered competing clinical diagnoses such as malaria, typhoid, viral hepatitis, and other febrile illnesses. ${ }^{23,39-57}$ More importantly, a majority of studies (82\%) confirmed suspected cases with laboratory testing, assuring reviewers that included patients were likely infected with dengue. This point further highlights ultrasound's potential to differentiate severe dengue from other febrile illnesses in which vascular leakage is not seen, although further research in this area is required.

Our review could have benefitted from an analysis of the type of laboratory studies used to identify and confirm cases to understand the pattern of use, accessibility, and feasibility of certain laboratory tests in settings where dengue is endemic. Moreover, as laboratory testing does not reliably detect dengue in some cases and does not aid in case classification, this analysis could further elucidate the utility of ultrasound as an adjunctive examination. This analysis was not pursued in this review as it did not immediately relate to ultrasound use in dengue. However, such work is needed to determine ultrasound's applicability in early diagnosis and classification in relation to the laboratory tests preferred in these settings. 
Box 1

Research gaps and needs in the use of ultrasound in dengue

1. Access to ultrasound services for dengue patients

2. Potential geographical, virological, and patient-related (i.e., age, pregnancy, and comorbidities) differences in ultrasound findings

3. Contribution of ultrasound in the differential diagnosis of febrile subjects in dengue-endemic areas

4. Usefulness of portable ultrasound to detect early vascular leakage in primary care and limited-resource settings

5. Standardized protocol for focused assessment of ultrasound in dengue patients

6. Adequate gold standard comparison to estimate sensitivity and specificity of ultrasound to detect vascular leakage

7. Level of expertise (training) required to accurately assess vascular leakage with ultrasound in dengue patients

8. Impact of implementation of routine ultrasound examination in patient correct classification, management, and prognosis

Studies with hospitalized patients or patients in the intensive care unit (i.e., patients likely to have severer forms of dengue), compared with patients in the ambulatory setting, dominate the current evidence on ultrasound use in dengue. As the utility of ultrasound in dengue lies in its capacity to inform early management decisions and avoid unnecessary hospitalizations, we consider the relative lack of studies in the ambulatory setting a gap in knowledge warranting further investigation.

Ultrasound examinations are traditionally administered by radiology departments in secondary or tertiary levels of care by request of the treating physician. Other medical specialists (e.g., emergency medicine, internal medicine, and obstetrics/ gynecology) can also provide the service directly or following a request from the treating physician but still limited to secondary or tertiary levels of care. Point-of-care ultrasound (POCUS) can be performed at the primary level of care, by primary care physicians, general practitioners, and, in some situations, nonmedical personnel (e.g., technicians), who perform ultrasound examinations and make clinical decisions based on their findings. ${ }^{58}$ Point-of-care ultrasound appears to be in limited use in dengue patients as portable ultrasound equipment and the performer being a primary care physician were relatively infrequent in included studies. Given ultrasound's potential to detect early vascular leakage, the usefulness of portable ultrasound in primary care settings to identify and triage severe cases of dengue deserves more substantial examination.

Timing of ultrasound examination is particularly important as it relates to the phases of dengue illness and the respective management in these phases. Patients can progress to the critical phase and develop plasma leakage as early as the third day of illness, requiring prompt hospitalization and fluid therapy. However, in our review, ultrasound examination was principally conducted on the first day of hospitalization, after presumed progression to the critical phase. This negates the utility of ultrasound as a predictive tool for the development of severe dengue, given these patients had likely already developed severe forms of dengue. Therefore, in our view, most studies conducted an ultrasound examination relatively late in the progression of dengue illness.

Ascites, pleural and pericardial effusion, and GBWT were the most common ultrasound findings in dengue. . $^{18,19,30,35,41,53,59-80}$ By contrast, only one study in this review found pulmonary B-lines suggestive of pulmonary edema. ${ }^{18}$ Given the $97 \%$ sensitivity of ultrasound for the diagnosis of pulmonary edema, this finding may suggest a relative dearth of vascular leakage into the pulmonary airspace and parenchyma in dengue as compared with the peritoneal cavity, thoracic cavity, pericardial sac, and gallbladder wall, or limited exploration of this sign. ${ }^{81}$ The minimal ultrasound evidence of pulmonary edema in dengue corresponds with findings from other studies using chest radiograph and computed tomography to study lung involvement. ${ }^{82,83}$

We did not identify a standard protocol to perform ultrasound in dengue patients as it has been standardized for trauma (Focused Assessment With Sonography for Trauma) and shock and hypotension (Rapid Ultrasound for Shock and Hypotension), among other conditions. ${ }^{84,85}$ Number and location of sites and periodicity of examinations were highly variable. Thorax was the most common anatomical site, but the specific anatomical exploration, technique, duration, and frequency were not specified. The same occurred with the abdomen, for which no standard cutoff point to define the GBWT was reported. Moreover, indications to perform cardiac and other site examinations were not clear. This affects the comparison between studies and conclusions about the frequency of ultrasound findings in dengue patients, as each study used an individualized, non-standardized approach to the ultrasound examination.

The limitations in the current available evidence to assess ultrasound's sensitivity and specificity to differentiate severe from non-severe dengue have been previously reported. ${ }^{9}$ In that systematic review, WHO 1997 and 2009 severity definitions were used as reference standards, but in our review, most studies did not report the severity definition used. We also looked for reference standards used for vascular leakage, but most studies did not report them either. This suggests the need to improve implementation of guidelines for reporting of diagnostic studies. ${ }^{86}$ We found one study that determined interrater reliability; this study reported good concordance (kappa index) and can be further replicated to evaluate ultrasound training interventions. ${ }^{35}$ Assessing the prognostic value of specific ultrasound findings in dengue patients is challenged by the fact that the findings are likely to influence management decisions. Hence, implementation of protocols of standardized focused ultrasound examination following cluster designs is more suitable to provide evidence on the usefulness and impact of ultrasound in dengue patients in different settings. 
Patient outcomes were described in less than a third of studies. This indicates a significant limitation in the current literature: low reporting of patient outcomes and, more importantly, no studies which determined whether ultrasound helps as an independent predictor of morbidity or mortality. Within the studies that did report patient outcomes, mortality was reported more than half the time, likely as a result of the predominance of hospitalized patients in our review.

Clinicians and policy-makers may consider the data presented here when implementing ultrasound in the routine clinical care of dengue patients. However, the evidence is still insufficient in relation to frequency, accuracy, and prognostic value of ultrasound findings in dengue patients. Nonetheless, the current portable technologies and multiple applications of ultrasound could be advantageous for its implementation and cost-benefit analysis. ${ }^{37}$ As with any other medical technology, training and assessment of competence is mandatory for proper implementation. ${ }^{87} \mathrm{~A}$ summary of research gaps and needs is provided in Box 1.

In conclusion, the present scoping review provides an overview of the primary literature on the use of ultrasound in dengue. The large aggregate of studies with ultrasound findings appears to support the use of ultrasound in dengue; however, further research is required to elucidate its precise diagnostic and prognostic value. Notably, ascites, pleural effusion, and GBWT are key markers of ultrasound detection of vascular leakage in dengue patients. A focused POCUS protocol for triaging dengue patients could be evaluated for its effectiveness in improving management, patient outcomes, and cost-benefit in the care of this expansive tropical disease.

Received February 6, 2020. Accepted for publication December 14, 2020.

Published online January 18, 2021.

Note: Supplemental Appendix appears at www.ajtmh.org.

Acknowledgments: We thank Christian Messu at Universidad del Valle for his contribution to initial screening and Melissa Eelkema at Hennepin County Medical Center for her contribution to the search of manuscripts.

Financial support: This work was supported by the following sources: the Doris Duke Charitable Foundation through a grant supporting the Doris Duke International Clinical Research Fellow Program at the University of Minnesota (N. D.); the University of Minnesota's Center for Global Health and Social Responsibility and the Fulbright U.S. Scholar Program (J. K.); and Universidad del Valle, Cali, Colombia (L. O., D. Z.).

Authors' addresses: Neelesh Dewan, University of Miami Miller School of Medicine, Miami, FL, E-mail: neeleshdewan92@gmail.com. Daniela Zuluaga and Lyda Osorio, Epidemiology and Population Health Research Group (GESP), School of Public Health, Universidad del Valle, Cali, Colombia, E-mails: dzuluaga1104@gmail.com and lyda.osorio@correounivalle.edu.co. Mary-Ellen Krienke, University of Minnesota Medical School, Minneapolis, MN, E-mail: krien021@ umn.edu. Caitlin Bakker, Health Sciences Libraries, University of Minnesota, Minneapolis, MN, E-mail: cjbakker@umn.edu. Jonathan Kirsch, Division of General Internal Medicine, University of Minnesota Medical School, Minneapolis, MN, E-mail: kirsch@umn.edu.

\section{REFERENCES}

1. Brady OJ, Gething PW, Bhatt S, Messina JP, Brownstein JS, Hoen AG, Moyes CL, Farlow AW, Scott TW, Hay SI, 2012. Refining the global spatial limits of dengue virus transmission by evidencebased consensus. PLoS Negl Trop Dis 6: e1760.
2. Bhatt $S$ et al., 2013. The global distribution and burden of dengue. Nature 496: 504-507.

3. World Health Organization, 2020. Dengue and severe Dengue. Geneva, Switzerland: WHO. Available at: https://www.who.int/ news-room/fact-sheets/detail/dengue-and-severe-dengue. Accessed October 22, 2020

4. World Health Organization, 2009. Dengue: Guidelines for Diagnosis, Treatment, Prevention and Control: New Edition. Geneva, Switzerland: WHO. Available at: http://www.ncbi.nlm.nih.gov/books/ NBK143157/. Accessed October 22, 2020.

5. Simmons CP, Farrar JJ, Nguyen van VC, Wills B, 2012. Dengue. N Engl J Med 366: 1423-1432.

6. Froudarakis ME, 2008. Diagnostic work-up of pleural effusions. Respir Int Rev Thorac Dis 75: 4-13.

7. de Oliveira RVB, Rios LTM, dos Remédios Freitas Carvalho Branco M, Lopes Braga Júnior L, Nascimento JMS, Silva GF, Bandeira KP, 2010. Valor da ultrassonografia em crianças com suspeita de febre hemorrágica do dengue: revisão da literatura. Radiol Bras 43: 401-407.

8. Rudralingam V, Footitt C, Layton B, 2017. Ascites matters. UItrasound Leeds Engl 25: 69-79.

9. Low GKK, Looi SY, Yong MH, Sharma D, 2018. Predictive and diagnostic test accuracy of ultrasonography in differentiating severe dengue from nonsevere dengue. J Vector Borne Dis 55: 79-88.

10. Ibrahim F, Thio THG, Faisal T, Neuman M, 2015. The application of biomedical engineering techniques to the diagnosis and management of tropical diseases: a review. Sensors 15: 6947-6995.

11. Dewan N, Messu C, Eelkema M, Osorio L, Bakker C, Kirsch J, 2019. A Scoping Review of the Use of Ultrasound in Dengue: Protocol for a Scoping Review. Available at: https://osf.io/ v7jqw/. Accessed February 7, 2019.

12. The EndNote Team, 2016, Endnote. Philadelphia, PA: Clarivate Analytics.

13. Rayyan QCRI [Internet], 2016. Doha, Qatar: Qatar Computing Research Institute. Available at: https://rayyan.qcri.org/ welcome. Accessed January 7, 2019.

14. Ouzzani M, Hammady H, Fedorowicz Z, Elmagarmid A, 2016. Rayyan-a web and mobile app for systematic reviews. Syst Rev 5: 210.

15. Harris PA, Taylor R, Thielke R, Payne J, Gonzalez N, Conde JG, 2009. Research electronic data capture (REDCap)--a metadatadriven methodology and workflow process for providing translational research informatics support. J Biomed Inform 42: 377-381.

16. Whiting PF, Rutjes AWS, Westwood ME, Mallett S, Deeks JJ, Reitsma JB, Leeflang MM, Sterne JA, Bossuyt PM; QUADAS-2 Group, 2011. QUADAS-2: a revised tool for the quality assessment of diagnostic accuracy studies. Ann Intern Med 155: 529-536.

17. Microsoft, 2020. Microsoft Excel for Mac. Redmond, WA: Microsoft Corporation.

18. Thanachartwet $V$, Wattanathum $A$, Sahassananda $D$, Wacharasint $P$, Chamnanchanunt S, Khine Kyaw E, Jittmittraphap A, Naksomphun M, Surabotsophon M, Desakorn V, 2016. Dynamic measurement of hemodynamic parameters and cardiac preload in adults with dengue: a prospective observational study. PLoS One 11: e0156135

19. Parmar JP, Mohan C, Vora M, 2017. Patterns of gall bladder wall thickening in dengue fever: a mirror of the severity of disease. Ultrasound Int Open 3: E76-E81.

20. Levaggi ND, Lucas AN, Barletta JA, 2017. Bilateral acute angle closure in a patient with dengue fever: a case report. Arq Bras Oftalmol 80: 266-267.

21. Setiawan MW, Samsi TK, Wulur H, Sugianto D, Pool TN, 1998. Dengue haemorrhagic fever: ultrasound as an aid to predict the severity of the disease. Pediatr Radiol 28: 1-4.

22. Setiawan MW, Samsi TK, Wulur H, Sugianto D, Pool TN, 1998. Epigastric pain and sonographic assessment of the pancreas in dengue hemorrhagic fever. J Clin Ultrasound 26: 257-259.

23. Shamim M, 2010. Frequency, pattern and management of acute abdomen in dengue fever in Karachi, Pakistan. Asian J Surg 33: 107-113. 
24. Sudulagunta SR, Sodalagunta MB, Sepehrar M, Bangalore Raja SK, Nataraju AS, Kumbhat M, Sathyanarayana D, Gummadi S, Burra HK, 2016. Dengue shock syndrome. Oxf Med Case Rep 2016: 269-272.

25. Weerakoon KGAD, Chandrasekaram S, Jayabahu JPSNK, Gunasena S, Kularatne SAM, 2009. Acute abdominal pain in dengue haemorrhagic fever: a study in Sri Lanka, 2009. Dengue Bull 33: 70-74.

26. Asghar J, Farooq K, 2011. Radiological appearance and their significance in the management of dengue hemorrhagic fever. Pak J Med Health Sci 5: 685-692.

27. González Cortiñas M, Vidal González D, Cepero Cordero J, Lashley Oliveras ML, 1999. Dengue hemorrágico. Estudio clínico de 200 pacientes. Rev Cuba Med 38: 13-18.

28. Martínez López JÁ, 2010. Caracterización clinicoepidemiológica y ecográfica de pacientes con dengue confirmado. Medisan 14: 665-674. Available at: http://bvs.sld.cu/revistas/san/vol_14_ 5_10/san11510.htm. Accessed February 28, 2019.

29. Viswanathan V, Andankar P, Gupta N, Singh I, Rajguru M, Laxmivenkateshaiah S, 2017. Simultaneous occurrence of Kawasaki disease and salmonella infection with a positive dengue serology. Pediatr Rheumatol 15: 175.

30. Gupta BK, Nehara HR, Parmar S, Meena SL, Gajraj S, Gupta J, 2017. Acute abdomen presentation in dengue fever during recent outbreak. J Acute Dis 6: 198-204.

31. Premaratna R, Bailey MS, Ratnasena BGN, de Silva HJ, 2007. Dengue fever mimicking acute appendicitis. Trans $R$ Soc Trop Med Hyg 101: 683-685.

32. Yousaf KR, Atiq S, Sheikh QS, Nisar MS, Mansoor Z, Khalid S, 2011. Sonographic features of polyserositis as an adjunct to clinico - pathological parameters in diagnosing and predicting the severity of dengue fever. Pak J Med Health Sci 5: 184-189.

33. Rao SV, Jacob GG, Raju NA, Ancheri S, 2016. Spontaneous arterial hemorrhage as a complication of dengue. Indian $J$ Crit Care Med 20: 302-304.

34. Available at: SciePro/Shutterstock.com.

35. Nainggolan L, Wiguna C, Hasan I, Dewiasty E, 2018. Gallbladder wall thickening for early detection of plasma leakage in dengue infected adult patients. Acta Med Indones 50: 193-199.

36. Goldberg BB, Goodman GA, Clearfield HR, 1970. Evaluation of ascites by ultrasound. Radiology 96: 15-22.

37. Lele PP, 1972. Application of ultrasound in medicine. $N$ Engl $J$ Med 286: 1317-1318.

38. Sippel S, Muruganandan K, Levine A, Shah S, 2011. Review article: use of ultrasound in the developing world. Int J Emerg Med 4: 72.

39. Arshad K, Sheikh S, Naqvi SUB, Sarwar I, Javaid S, Asghar M, Butt MA, 2015. Frequency of splenomegaly in dengue fever in children. J Ayub Med Coll Abbottabad 27: 356-359.

40. Bhasin A, Kumar R, Chandra K, Singal RK, 2013. Dengue fever with myocarditis. J Indian Acad Clin Med 14: 187-189.

41. Byopadhyay D, Chattaraj S, Hajra A, Mukhopadhyay S, Ganesan $V, 2016$. A study on spectrum of hepatobiliary dysfunctions and pattern of liver involvement in dengue infection. $J$ Clin Diagn Res 10: OC21-OC26.

42. Chacko B, Subramanian G, 2008. Clinical, laboratory and radiological parameters in children with dengue fever and predictive factors for dengue shock syndrome. J Trop Pediatr 54: 137-140.

43. Coton T, Debonne JM, Molinier S, Chaudier B, Gras C, Carré D, Raillat A, 1999. Alithiasic cholecystitis and hemorrhagic dengue. Cholecystite Alithiasique Dengue Hemorragique 23: 789-790.

44. Gogna A, Pathak S, Dhamija K, Jharyan P, Arora BS, 2015. Changing clinical profile of dengue fever in Delhi in 2011. J Indian Acad Clin Med 16: 20-26.

45. Hernez JN, 2015. Case report: acute myocarditis cause death in dengue patient. Am J Trop Med Hyg 93: 434.

46. Kalenahalli J, Puja J, Vaddambal GM, Lingappa U, 2012. Hepatic involvement in dengue fever in children. Iran $J$ Pediatr 22: 231-236.

47. Kuna A, Wroczynska A, Gajewski M, Felczak-Korzybska I, Nahorski WL, 2016. A case of acalculous cholecystitis in the course of dengue fever in a traveller returned from Brazil. Int Marit Health 67: 38-41.

48. Marín J, Vilcarromero S, Forshey BM, Celis-Salinas JC, RamalAsayag C, Morrison AC, Laguna-Torres A, Casapía M, Halsey ES, 2013. Compromiso gastrointestinal agudo en pacientes con dengue por serotipo 4: comunicación de un caso y revisión de la literatura. Rev Chil Infectol 30: 541-547.

49. Meena K, Kumar P, Anita, Paul P, 2013. Acute glomerulonephritis in dengue hemorrhagic fever: a rare case report. Ann Trop Med Public Health 6: 581-582.

50. Meltzer E, Heyman Z, Bin H, Schwartz E, 2012. Capillary leakage in travelers with dengue infection: implications for pathogenesis. Am J Trop Med Hyg 86: 536-539.

51. Muhammad S, Junaid R, Muneeza N, Yaseen A, Ayesha A, 2016. Clinical spectrum of dengue fever in pediatric age at a tertiary care hospital in Lahore. Esculapio 12: 126-129.

52. Rueda E, Méndez A, González G, 2002. Hemophagocytic syndrome associated with dengue hemorrhagic fever. Bioméd Rev Inst Nac Salud 22: 160-166.

53. Seervi N, Meena SR, Saluja M, Meena SB, Shringi P, Saini PK, 2017. Hepatic dysfunction in patients of dengue viral infection in Kota region. J Indian Acad Clin Med 18: 18-21.

54. Sehgal A, Gupta S, Tyagi V, Bahl S, Singh SK, Puliyel JM, 2002. Gall bladder wall edema is not pathogenic of dengue infection. J Trop Pediatr 48: 315-316.

55. Shah S, Rolfe R, Henostroza G, Seas C, 2018. Images in clinical tropical medicine: ultrasound findings of plasma leakage in dengue fever. Am J Trop Med Hyg 99: 1362-1363.

56. Tahir H, Daruwalla V, Hayat S, 2015. Myocarditis leading to severe dilated cardiomyopathy in a patient with dengue fever. Case Rep Cardiol 2015: 319312.

57. Tee TJ, 2015. Dengue infection with severe acute hepatitis and acalculus cholecystitis: a diagnostic challenge. Am J Gastroenterol 110: S356.

58. The Society of Point of Care Ultrasound, 2017. Guidelines For Point Of Care Ultrasound Utilization In Clinical Practice. Available at: https://spocus.org/wp-content/uploads/2019/12/GUIDELINESFOR-POINT-OF-CARE-ULTRASOUND-UTILIZATION-INCLINICAL-PRACTICE.pdf. Accessed October 24, 2020.

59. Dayana Kumar KR, Halawar RS, 2018. Comparative study of ultrasound findings in seropositive pediatric and adult patients with dengue fever. Radiol Infect Dis 5: 59-62.

60. Kularatne SAM, Rajapakse MM, Ralapanawa U, Waduge R, Pathirage LPMMK, Rajapakse RPVJ, 2018. Heart and liver are infected in fatal cases of dengue: three PCR based case studies 11 medical and health sciences 1103 clinical sciences. BMC Infect Dis 18: 681.

61. Ferreira BDC, Correia D, 2018. Ultrasound assessment of hepatobiliary and splenic changes in patients with dengue and warning signs during the acute and recovery phases. $J$ Ultrasound Med 38: 2015-2024.

62. Nanjundappa RC, Bc VC, 2018. Predicting the severity of dengue fever in children based on ultrasound, peripheral venous lactate and proteinuria. Pediatr Crit Care Med 19: 111-112.

63. Rabbani MU, Aslam M, Zaheer MS, Ashraf MU, 2018. Clinical and laboratory profile of dengue fever in a north Indian tertiary hospital. J Assoc Physicians India 66: 37-39.

64. Bhargava S, Srivastava S, Gupta A, 2017. Ultrasonographic findings in dengue fever: a retrospective study. J Int Med Sci Acad 30: 75-78.

65. Jayasundara B, Perera L, de Silva A, 2017. Dengue fever may mislead the surgeons when it presents as an acute abdomen. Asian Pac J Trop Med 10: 15-19.

66. Parmar J, Mohan C, Prem Kumar G, Vora M, 2017. Ultrasound is not useful as a screening tool for dengue fever. Pol $J$ Radiol 82: 693-700.

67. Ramabhatta S, Palaniappan S, Hanumantharayappa N, Begum SV, 2017. The clinical and serological profile of pediatric dengue. Indian J Pediatr 84: 897-901.

68. Chak S, Kumar A, 2016. Can radiology play a role in early diagnosis of dengue fever? North Am J Med Sci 8: 100-105.

69. Acharyya A, Ghosh K, Bhattacharyya A, Ghosh M, Chakraborty S, Ghosh S, Pal M, 2016. The dengue fever and its complication: a 
scenario in a tertiary-level hospital of greater Kolkata. Ann Trop Med Public Health 9: 92-96.

70. Agarwal N, Jain $P, 2016$. Sonography in dengue fever: an adjunct to clinico-laboratory profile. Indian J Public Health Res Dev 7: 299-303.

71. Ferno S, Wijewickrama A, Gomes L, Punchihewa CT, Madusanka $\mathrm{SD}$, Dissanayake H, Jeewandara C, Peiris H, Ogg GS, Malavige GN, 2016. Patterns and causes of liver involvement in acute dengue infection. BMC Infect Dis 16: 319.

72. Khurram M, Qayyum W, Umar M, Jawad M, Mumtaz S, Khaar HTB, 2016. Ultrasonographic pattern of plasma leak in dengue haemorrhagic fever. J Pak Med Assoc 66: 260-264.

73. Kularatne SAM, Ralapanawa U, Dalugama C, Jayasinghe J, Rupasinghe S, Kumarihamy P, 2018. Series of 10 dengue fever cases with unusual presentations and complications in Sri Lanka: a single centre experience in 2016. BMC Infect Dis 18: 674.

74. Laoprasopwattana $\mathrm{K}, 2016$. Thrombocytopenia is the most important predictive factor of plasma leakage and correlates with plasma leakage severity. Eur J Pediatr 175: 1494.

75. Thein TL et al., 2014. Ultrasound and chest X-ray in an adult patient with dengue. Am J Trop Med Hyg 91: 321.

76. Malavige GN et al., 2016. Efficacy of rupatadine in the treatment of acute dengue infection. Am J Trop Med Hyg 95: 34.

77. Pothapregada S, Kullu P, Kamalakannan B, Thulasingam M, 2016. Is ultrasound a useful tool to predict severe dengue infection? Indian J Pediatr 83: 500-504.

78. Jain D, Rajput $R$, Pathak V, Mittal A, Jain $P, 2017$. Changing trends in clinical presentation and biochemical spectrum of dengue fever: an observation of a tertiary care centre. Arch Clin Infect Dis 12: e62221.
79. Malladi SVS, Gone V, Adiraju KP, Chand N, Yadati SR, 2016. Clinical, laboratory profile and outcome of patients with dengue viral infection at a south Indian tertiary care hospital. Int J Infect Dis 45: 445.

80. Mondal R, Pemola Devi N, Jauhari RK, 2016. Studies on symptomatic profiles of dengue fever (DF) vis-à-vis non-dengue fever (NDF) in district Dehradun, uttarakhand. J Commun Dis 48: 15-20.

81. Wang Y, Shen Z, Lu X, Zhen Y, Li H, 2018. Sensitivity and specificity of ultrasound for the diagnosis of acute pulmonary edema: a systematic review and meta-analysis. Med Ultrason 1: 32-36.

82. Wang CC, Wu CC, Liu JW, Lin AS, Liu SF, Chung YH, Su MC, Lee IK, Lin MC, 2007. Chest radiographic presentation in patients with dengue hemorrhagic fever. Am J Trop Med Hyg 77: 291-296.

83. Rodrigues RS, Brum ALG, Paes MV, Póvoa TF, Basilio-deOliveira CA, Marchiori E, Borghi DP, Ramos GV, Bozza FA, 2014. Lung in dengue: computed tomography findings. PLoS One 9: e96313.

84. American Institute of Ultrasound in Medicine, 2014. Focused Assessment with Sonography for Trauma (FAST) Examination. Available at: https://www.aium.org/resources/guidelines/fast. pdf. Accessed January 8, 2020.

85. Seif D, Perera P, Mailhot T, Riley D, Mandavia D, 2012. Bedside ultrasound in resuscitation and the rapid ultrasound in shock protocol. Crit Care Res Pract 2012: 503254

86. Cohen JF et al., 2016. STARD 2015 guidelines for reporting diagnostic accuracy studies: explanation and elaboration. BMJ Open 6: e012799.

87. Kurjak A, Breyer B, 1986. The use of ultrasound in developing countries. Ultrasound Med Biol 12: 611-621. 